\title{
RESEARCH ON DC-CLEARING ELECTRIC FIELD AND CHANGES OF FOCUSING STRUCTURE PARAMETERS OF STORAGE RING
}

\author{
X.Q. Wang ${ }^{*}$, Y.J. Pei, X.L. Dong, H.L. Xu, L. Shang, L. Wang, \\ K.J. Fan, G.C. Wang, C.G. Yao, S.M. Hu, X.F. Luo \\ NSRL, USTC, P.O. Box 6022, Hefei, Anhui 230029, China
}

\begin{abstract}
This paper presents DC clearing field to clear ions in vacuum pipe of Hefei ring. It also presents changes of focusing structure parameters caused by the clearing field. It concludes that tune shifts caused by the field are related to real-time close orbit of the beam. The paper points out that the field asymmetrically distributed along the ring destroys the symmetry of focusing structure and decreases the ring acceptance, which has negative effect to injection and accumulation process in certain condition.
\end{abstract}

\section{INTRODUCTION}

Some clearing field patterns to suppress instability are reported by the papers ${ }^{1-4}$. One of them is produced by DCclearing electrode in circular tube, its function was described $^{5-8}$, but the potential reported in paper 9 and 10 isn't analytic in two-dimension boundary problem. The force and potential functions presented in this paper are analytic; the data are taken from Hefei ring. There are thirteen electrodes in the tubes installed asymmetrically along the ring, their length is 17.9 meters that is 1.86 times the length of all quadrupole magnets. They have played an important role in clearing ion, suppressing ion instability and storing beam since $1989^{5-8,11,12}$. These electrodes destroy the lattice symmetry, research on linear influence of the field to betatron tunes of beam was presented $^{1-4,9}$. This paper only discusses the influence of the dipole and quadrupole of DC clearing field on beam: Twiss parameters ${ }^{13}$, ring acceptance and multi turn injection.

\section{ANALYTIC EXPRESSIONS}

Analyze the DC field in unaxial bicylindrical surfaceboundary, the analytic expressions of potential and field caused by inner wire in circular pipe ${ }^{11}$ can be get,

$$
\begin{aligned}
& \varphi(x, z)=-\frac{V_{d c e}}{\ln (a r / R \delta)} \cdot \ln \left[\frac{R \sqrt{x^{2}+(z+a)^{2}}}{a \sqrt{x^{2}+(z+b)^{2}}}\right] \\
& \psi_{+}(x, z)=-\frac{V_{d c e}}{\ln (a r / R \delta)} \cdot \operatorname{arctg}\left[\frac{x^{2}+z^{2}+z(b+a)+R^{2}}{x(b-a)}\right](\mathrm{x}>0) \\
& \psi_{-}(x, z)=+\frac{\pi V_{d c e}}{\ln (a r / R \delta)}-\psi_{+}(-x, z) \quad(\mathrm{x}<0) \\
& a=a_{0}+\delta, \quad b=\frac{R^{2}}{a_{0}+\delta}
\end{aligned}
$$

\footnotetext{
*wangxaqi@ustc.edu.cn, xwang@ssrl.slac.stanford.edu (only before June 3, 1999)
}

$$
\delta=\frac{2 a_{0} R^{2}}{R^{2}+a_{0}^{2}-r^{2}+\sqrt{\left(R^{2}+a_{0}^{2}-r^{2}\right)^{2}-4 a_{0}^{2} R^{2}}}-a_{0}
$$

Here, the coordinate origin is on pipe axis. $x$ is radial coordinate (horizontal on the cross section). $z$ is vertical. $R$ is inner radius of the pipe, and $r$ is radius of the wire. $a_{0}$ is the distance from the wire to the pipe axis, and $V_{d c e}$ is DC clearing voltage. The unit of $V_{d c e}$ is DC clearing voltage and other units are meter. The twodimension schematic diagrams of the clearing field are shown in Fig.1 for an example. The figure presents equipotential lines and field lines.
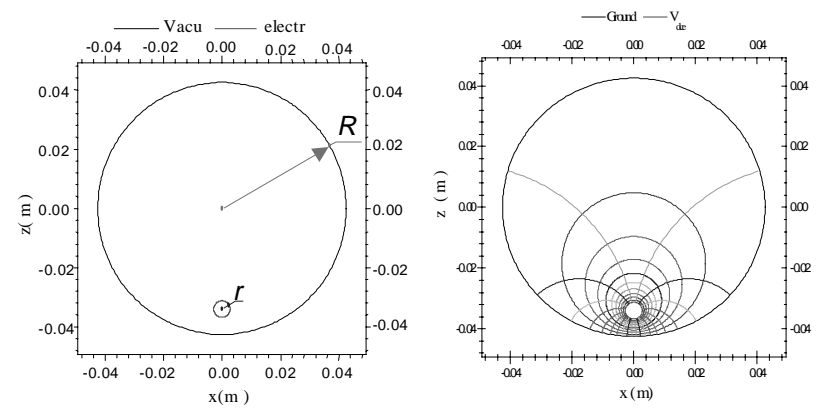

Fig.1 Schematic diagram of equipotential lines and field lines of the DC clearing field

\section{TUNE SHIFTS NEAR AXIS OF PIPE}

There is focusing effect due to quadrupole of the field ${ }^{11}$.

\subsection{Linear formulation}

The focusing effect of the field quadrupoles is

$$
K_{E}=K_{z}(0,0)=-K_{x}(0,0)=\frac{-e\left(b^{2}-a^{2}\right)}{E_{C} R^{4} \ln (a r / R \delta)} \cdot V_{d c e}
$$

in first-order approximation. The unit of $K_{E}$ is $\mathrm{m}^{-2}, E_{c}$ is $\mathrm{eV}$ and $e$ is an electronic charge. So the quardupole of field is focusing (defocusing) on beam in vertical (horizontal), and second-order differential equations are

$$
\begin{aligned}
& \frac{d^{2} x}{d s^{2}}+\left(K_{Q}-K_{E}\right) x=0, \\
& \frac{d^{2} z}{d s^{2}}-\left(K_{Q}-K_{E}\right) z=0 .
\end{aligned}
$$

Here, the unit of $K_{Q}$, focusing strength of quadrupole magnets, is $\mathrm{m}^{-2}$. The $K_{E}$ is always less than $K_{Q}$. Thus, the tune shifts of beam are 


$$
\begin{aligned}
& \Delta v_{x}=\frac{e\left(b^{2}-a^{2}\right) \int_{L_{d c e}} \beta_{x}(s) d s}{4 \pi E_{C} R^{4} \ln \left(\frac{a r}{R \delta}\right)} \cdot V_{d c e}, \\
& \Delta v_{z}=-\frac{e\left(b^{2}-a^{2}\right) \int_{L_{d c e}} \beta_{z}(s) d s}{4 \pi E_{C} R^{4} \ln \left(\frac{a r}{R \delta}\right)} \cdot V_{d c e}
\end{aligned} .
$$

$L_{\text {dce }}$ implies that integrates in the clearing field sections. It's obvious that tunes are linear relevance to voltage. The coefficients rely only upon the lattice mode and the electrode structure, and their sign reverse in different direction. The relevance is illustrated in fig. 2 and fig. 3 (circle dot is calculated, square dot is measured). The horizontal coordinates show applied voltage and the vertical show tune. Here, the straight line segments being
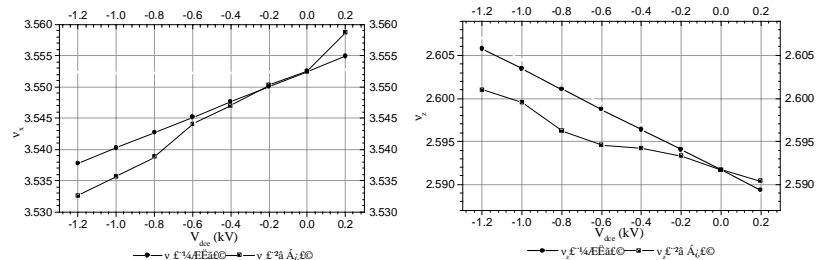

Fig.2 Schematic change Fig.3 Schematic change of tune in hori. Direction of tune in verti. Direction

approximately beeline is based on the (4), and another line segments is based on measured data. We have three conclusions from the figures. First, there is defocusing in vertical and focusing in horizontal based on the (4) or the measured data. Second, the tune shifts always increase as the voltage rises based on numerical calculation or the measured data. Third, relevance between the tune shifts and voltage is nonlinear based on measured data, which are different from the (4).

\subsection{Nonlinear formulation}

Fig. 2 and 3 show that tune shift is larger in vertical than in horizontal at the same DC voltage. In linear formulation, the effect of the quadrupoles of field on the beam is calculated along the pipe axis. Neglect higher order quantities such as $(x / a)^{2},(x / b)^{2},(z / a)^{2}$ and $(z / b)^{2}$ in the denominators, we can derive second-order nonlinear differential equations,

$$
\begin{aligned}
& \frac{d^{2} x}{d s^{2}}+\left[K_{Q}-K_{E}\left(1-2 \cdot \frac{a^{2}+a b+b^{2}}{R^{2}(a+b)} z\right)\right] x=0, \\
& \frac{d^{2} z}{d s^{2}}-\left(K_{Q}-K_{E}\right) z=-\frac{e V_{d c e}(b-a)}{E_{C} R^{2} \ln (a r / R \delta)} .
\end{aligned}
$$

\subsection{Relevance on close orbit}

The right of (5b) is an analogue of close orbit displacement. There is always a vertical dipole of the field on the vertical symmetry plane ${ }^{11,12}$, so the stored beam leave from the central orbit, that is why the real focusing strength is smaller than $K_{E}$ based on (4). The displacement between close orbits is shown in fig.4, which is respectively with or without DC-septum magnetic field (electrons energy is $200 \mathrm{MeV}$ or $800 \mathrm{MeV}$ ), the DC voltage is $-1 \mathrm{kV}$, the baseline (square dot) is the beam close orbit (energy of $800 \mathrm{MeV}$ ) without DC-septum magnetic field. The horizontal axes denote the longitudinal coordinate of the ring. The difference among the real orbits can be observed obviously in the fig.4.

B. Autin defined tune is the mean value of betatron oscillations per turn over a great number of turns ${ }^{14}$. The differential equation (5b) contains the correction term related to close orbit, the correction term sign indicates vertical orientation of close orbit. Thus, we may calculate accurately tune shift so long as we know close orbit along ring. But, it's only a rough calculation if without enough accuracy of monitor system.

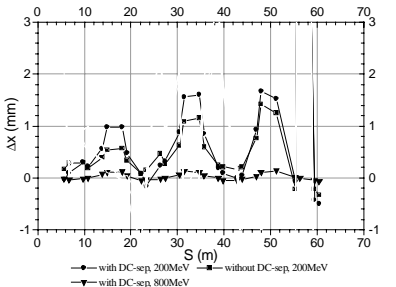

(a) hori. close orbits

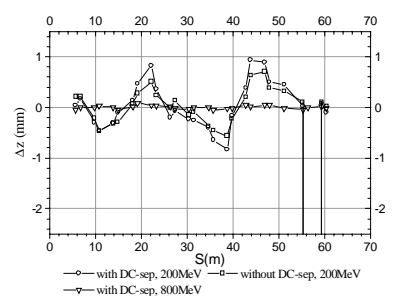

(b) verti. close orbits
Fig.4 The relative displacement measured

The tune shifts change obviously as close orbit moves based on the nonlinear formulation. The vacuum chamber of Hefei ring was opened at the first of 1997. When it was closed, the injection working point changed and the accumulation capability was bad when a couple of electrodes didn't operate, the machine runs well after all electrodes operated again.

\section{CORRECTION OF RING LATTICE}

Fig.5 shows the fitting curves of $\beta$ and $\eta$ functions of the GPLS mode without clearing field, the starts of curves are the midpoint of fourth long linear section.

\section{1 asymmetric distribution}

Regarding whole ring as one period, by the help of MULIN code (developed in the NSRL), the $\beta$ and $\eta$ functions of the GPLS mode with DC-clearing field can be calculated, the DC voltage is $-1.0 \mathrm{kV}$, and other parameters are the same as those without clearing field. There are three cases: first, all of thirteen electrodes operate; next, two of them turn off; last; fifteen electrodes operate after Phase two project will been completed. These $\beta$ and $\eta$ functions are shown in the fig.6, 7 and 8 . Obviously, the symmetry of GPLS mode is distorted and $\beta$ and $\eta$ functions become asymmetry along the ring as the electrodes operate in the fig.6 and 7. If fifteen electrodes are in operation, the symmetry of GPLS mode will approach those without clearing electrode. 

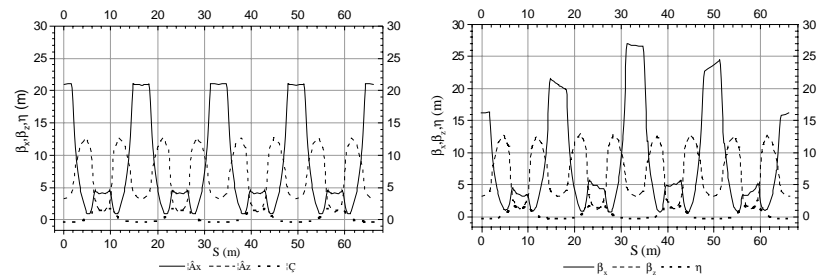

5 , without electrode.

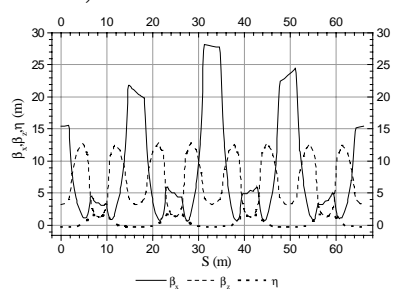

7, with 13 electrodes.
6 , with 11 electrodes.

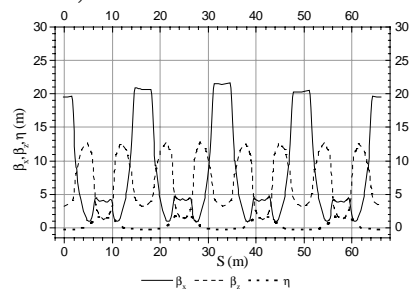

8 , with 15 electrodes.

Fig.5-8 Twiss parameter curves along the ring

\subsection{Ring acceptance}

The $\beta_{x}$ distribution in each long linear section is same without the electrode. When electrodes work, the $\beta_{x}$ in the fourth long linear section decreases while increases in the second long linear section, see Fig.6 and 7. The $\beta_{x}$ in the second section midpoint is larger than that of the first one, their ratios are 1.31 in Fig.6 and 1.37 in Fig.7, that means the amplitude of betatron oscillation increases respectively $15 \%$ and $17 \%$, in other words, the ring acceptances decrease. Simply, neglect energy spread and consider the dynamic aperture is large enough, study the influence of clearing field on ring acceptance. Without electrode, the injection bottleneck of GPLS mode is the restrict of the strip of pulse septum near injection point, the acceptance $81 \mathrm{~nm} \cdot \mathrm{rad}$. The bottleneck maybe in the second long section when electrods work, the acceptance is $66 \mathrm{~nm} \cdot \mathrm{rad}$, that means the acceptance will decrease $19 \%$ if DC electrodes in operation asymmetrically.

It will increases commissioning complicacy if the vacuum chamber aperture in second straight section decreases. The horizontal half width of the chamber is 40 $\mathrm{mm}$ after superconducting wiggler was installed. The ring acceptance decreases $30 \%$, down to $57 \mathrm{~nm} \cdot \mathrm{rad}$ when the electrodes work. If 15 electrodes are in operation, The acceptance of the GPLS is $75 \mathrm{~nm} \cdot \mathrm{rad}$, only decrease $7 \%$, the limit of ring acceptance is the effective horizontal half width near the septum strip, and the acceptance will restore to $79 \mathrm{~nm} \cdot \mathrm{rad}$ if the chamber width remains its size.

Deduce from this, the double symmetry of HBLS mode is aloso destroied when the electrodes work. So the beam envelop becomes larger where $\beta$ function increases abnormally, the electrons lose easily and the accumulation becomes $\mathrm{s}$ difficult, that's why the commissioning of the HBLS mode is so difflcult now. The symmetry of the HBLS mode will be recovered and beam accumulation will be improved after fifteen DC clearing electrodes work.

\section{CONCLUSION}

Three conclusions can be drawn. First, the clearing electrodes can cause tune shifts. Second, the relativity between tune shifts and DC voltage is linear and can be calculated if no perturbation of closed orbit. Tune shifts will be affected by real-time closed orbit if the orbit is distorted, and can be calculated only based on nonlinear the asymmetrically distributed electrodes can destroy the periodic symmetry of Twiss parameters and decrease ring acceptance, which will do some bad effect on injection. If these electrodes are distributed symmetrically, these bad effects will decrease. Therefor, clearing electrodes should be installed symmetrically as possible.

\section{Reference}

[1] R D Kohaupt, In. Bericht DESY H1-71/2, 1971.

[2] T Kasuga et al., IEEE NS-32 (5) (1985) 2550.

[3] T Kasuga et al., J. Jour. of A. Phys. 24 (9) (1985) 1212.

[4] T Kasuga, J. Jour. of A. Phys. 25(11) (1986) 1711.

[5] Duohui He, Int. Jour. Mod. Phys. A(P. S.) 2A (1993) 552.

[6] Y J Pei, Proc. of $1^{\text {st }}$ Taiwan Straits Symp. on SR, 1993, p.40.

[7] C Y Yao \& Y J Pei, AIP Confer. Proc. 326, 1993, p.197.

[8] Y J Pei, J. of China of S. and T. 25 (1995) 64.

[9] L Chen et al., Proc. of the Workshop on Beam Ins. in Storage Rings, 1994, p.65.

[10] L Chen et al., Jour. of Shenzhen Uni. (S. \& E.) 13 (1 2) (1996) 1.

[11] X Q Wang et al., Jour. of China Uni. of S. \& T. 27 (2) (1997) 228.

[12] X Q Wang et al., Jour. of China Uni. of S. \& T. 28 (2) (1998) 166.

[13] E D Courant \& H S Snyder, Annals of Physics 3 (1958) 1.

[14] B Autin, AIP Confer. Proc. 153(1), 1985, p.288. 